\title{
Grand challenges in bone endocrinology
}

\section{Jon Tobias*}

Musculoskeletal Research Unit, School of Clinical Sciences, University of Bristol, Bristol, UK

*Correspondence: jon.tobias@bristol.ac.uk

This article gives a personal view of some of the major unanswered research questions at the interface between bone and endocrinology. One of the most compelling challenges relates to the emerging relationship between bone and energy metabolism. On a clinical level, it is well established that obesity exerts a protective effect on bone mass and risk of osteoporosis, an influence which appears to be established in childhood (Tobias, 2010). In terms of explaining this relationship between fat and bone, a series of studies by Karsenty and colleagues based on transgenic mice identified an intriguing pathway linking bone turnover and energy metabolism involving decarboxylated osteocalcin and insulin (Ferron et al., 2010). This finding is consistent with an inverse association between osteocalcin and insulin levels observed in elderly men (Kindblom et al., 2009). However, extraskeletal receptors for osteocalcin responsible for mediating such a pathway are yet to be identified. Moreover, there may be fundamental differences in the regulation of energy metabolism between man and rodent models, and in the absence of prospective clinical data linking changes in bone turnover to alterations in glucose tolerance and/or fat mass, the relevance of this mechanism to humans remains unclear.

A related question is whether bone metabolism is related to energy metabolism not only as a consequence of interactions with insulin, but also whether there is significant interplay with endocrine pathways originating from the intestine. It has been recognized for some time that feeding causes rapid suppression of bone markers such as beta CTX (Clowes et al., 2002), presumably reflecting an influence of gut-derived hormones on bone turnover. Intriguingly, further recent work by Karsenty and colleagues suggests that gut-derived hormones also exert a profound influence on bone mass, based on their findings in transgenic mice which suggest that reduced production of serotonin by the gut underlies familial high bone mass in patients with mutations of the LRP5 gene (Yadav et al., 2008), which in turn suggests that serotonin antagonists represent a novel approach to treating osteoporosis (Yadav et al., 2010). However, the role of reduced serotonin levels in mediating this phenotype remains controversial, not the least because patients with carcinoid syndrome, in whom circulating levels of serotonin are greatly increased, are not known to develop osteoporosis.

Dissecting out the mechanism of action of PTH, the major endocrine regulator of calcium metabolism, is a further important challenge. Continuous and intermittent exposure to PTH have diverse effects on bone, predominantly stimulating bone resorption and formation respectively. There have been extensive studies to characterize the intracellular signaling and downstream paracrine pathways involved in these actions (Datta and Abou-Samra, 2009), in the hope that this will enable the development of new anabolic therapies for osteoporosis that unlike teriparatide (which shares the 1-34 n-terminal of PTH and is administered by daily sc injection) can be given orally. Several paracrine factors have being identified which are also involved in the local actions of PTH on bone, such as the RANK/RANKL/OPG system involved in regulating osteoclast differentiation, and sclerostin which has been implicated in PTH-dependent effects on the osteoblast lineage (Kramer et al., 2010). Assays for these paracrine factors have revealed the presence of significant levels in the circulation, leading to the question of whether RANK, RANKL, OPG, and sclerostin represent endocrine regulators of bone in their own right. For example, recent studies suggest that the protective effect of estrogen on bone mass is associated with alterations in circulating levels of sclerostin (Modder et al., 2010). However, it is difficult to infer causality from these studies, and whether systemic levels of these factors as distinct to their local levels in bone have any meaningful regulatory role remains an important but as yet unanswered question.
A further interesting challenge is to dissect out the role of early life factors in bone development. There is intriguing evidence that early life factors particularly those operating in utero exert long term effects on subsequent growth and development through a process known as programming. Though most of this research has focused on relationships between in utero deprivation and outcomes related to other chronic conditions such as obesity, type II diabetes and heart disease, skeletal development may also be affected. Precisely which in utero factors are most important in this regard remains unknown, but possibilities include maternal vitamin D status (Javaid et al., 2006), which if confirmed point to simple interventions in the mother which may have important long term effects on skeletal health of the child.

There are also exciting opportunities for applying recent methodological advances to the study of bone endocrinology. Whereas the development of cloning and transgenic techniques nearly two decades ago led to major advances in elucidating a wide range of biological pathways, recent advances in genetic epidemiology have the potential to yield equally important insights. For example, genome wide association studies have not only confirmed associations between bone mineral density (BMD) and genetic markers in key bone regulatory genes such as OPG, RANK and RANKL, but have also identified an array of new genes involved in regulating bone mass (Rivadeneira et al., 2009). Individual genetic influences identified from these studies have been relatively small, and the majority of heritability of bone mass remains to be explained. Identifying this 'missing heritability' is a major challenge, and may depend on the study of rarer variants (requiring denser coverage of genome wide scans, application of whole genome sequencing and use of larger cohorts) and parent of origin effects (necessitating cohorts with DNA from successive generations). 
Whereas genetic studies in bone have largely been restricted to using BMD as measured by DXA as the main phenotypic outcome, this is a composite measure combining several different characteristics including bone size, cortical thickness, cortical density and the amount of trabecular bone. Use of alternative techniques such as PQCT which provide separate measures of these constituents may enable new genetic influences on bone to be identified, as illustrated by our recent finding of an independent signal within the RANKL gene associated with cortical BMD (Paternoster et al., in press). Characterizing genetic influences on bone in more detail may be particularly informative in terms of identifying factors that affect bone fragility and risk of fracture, given the important contribution of such parameters to overall bone strength. Although pQCT has limited resolution, newer techniques like extreme CT provide the opportunity for dissecting out genetic influences on further characteristics such as cortical porosity and trabecular number which may have relatively strong predictive value for fracture.

In summary, bone endocrinology faces several important challenges including confirmation that the skeleton plays a physiological role in regulating other systems such as energy metabolism, and that local factors such as sclerostin serve as endocrine regulators of bone. Recent methodological advances in areas such as genetics and the assessment of bone phenotypes provide new important opportunities for addressing these and related questions. With the disease burden due to osteoporotic fractures increasing rapidly as a result of the aging population, the ultimate challenge will be to translate these insights into new therapies which are superior to those in current use in terms of effectiveness, tolerability, safety and cost.

\section{REFERENCES}

Clowes, J. A., Hannon, R. A., Yap, T. S., Hoyle, N. R., Blumsohn,A., and Eastell, R. (2002). Effect of feeding on bone turnover markers and its impact on biological variability of measurements. Bone 30, 886-890.

Datta, N. S., and Abou-Samra, A. B. (2009). PTH and PTHrP signaling in osteoblasts. Cell. Signal. 21, 1245-1254.

Ferron, M., Wei, J., Yoshizawa, T., Del Fattore, A., DePinho, R. A., Teti, A., Ducy, P., and Karsenty, G. (2010). Insulin signaling in osteoblasts integrates bone remodeling and energy metabolism. Cell 142, 296-308.

Javaid, M. K., Crozier, S. R., Harvey, N. C., Gale, C. R., Dennison, E. M., Boucher, B. J., Arden, N. K., Godfrey, K. M., and Cooper, C. (2006). Maternal vitamin D status during pregnancy and childhood bone mass at age 9 years: a longitudinal study. Lancet 367, 36-43.

Kindblom, J. M., Ohlsson, C., Ljunggren, O., Karlsson, M. K., Tivesten, A., Smith, U., and Mellstrom, D. (2009). Plasma osteocalcin is inversely related to fat mass and plasma glucose in elderly Swedish men. J. Bone Miner. Res. 24, 785-791.

Kramer, I., Keller, H., Leupin, O., and Kneissel, M. (2010). Does osteocytic SOST suppression mediate PTHbone anabolism? Trends Endocrinol. Metab. 21, 237-244.

Modder, U. I., Clowes, J. A., Hoey, K., Peterson, J. M. McCready, L., Oursler, M. J., Riggs, B. L., and Khosla, S. (2010). Regulation of circulating sclerostin levels by sex steroids in women and in men. J. Bone Miner. Res. doi: 10.1002/jbmr.128. [Epub ahead of print].

Paternoster, L., Lorentzon, M., Vandenput, L., Karlsson, M. K., Ljunggren, O., Kindmark, A., Mellstrom, D., Kemp, J., Jarette, C. E., Holly, J., Sayers, A., St Pourcain, B., Timpson, N., Deloukas, P., Davey Smith, G., Ring, S., Evans, C., Tobias, J. H., and Ohlsson, C. (in press). Genome-wide association meta-analysis for cortical bone mineral density unravels allelic heterogeneity at the RANKL locus and potential pleiotropic effects on bone. PLoS Genet.

Rivadeneira, F., Styrkarsdottir, U., Estrada, K., Halldorsson, B. V., Hsu, Y.H., Richards, J. B., Zillikens, M. C., Kavvoura, F. K., Amin, N., Aulchenko, Y. S., Cupples, L.A., Deloukas, P., Demissie, S., Grundberg, E., Hofman, A., Kong, A., Karasik, D., van Meurs, J. B., Oostra, B., Pastinen, T., Pols, H. A., Sigurdsson, G., Soranzo, N., Thorleifsson, G., Thorsteinsdottir, U., Williams, F. M., Wilson, S. G., Zhou, Y., Ralston, S. H., van Duijn, C. M., Spector, T., Kiel, D. P., Stefansson, K., Ioannidis, J. P., and Uitterlinden, A. G. (2009). Twenty bone-mineral-density loci identified by large-scale meta-analysis of genome-wide association studies. Nat. Genet. 41, 1199-1206.

Tobias, J. H. (2010). Fat mass and bone development. Expert Rev. Endocrinol. Metab. 5, 323-325.

Yadav, V. K., Balaji, S., Suresh, P. S., Liu, X. S., Lu, X., Li, Z., Guo, X. E., Mann, J. J., Balapure, A. K., Gershon, M. D., Medhamurthy, R., Vidal, M., Karsenty, G., and Ducy, P. (2010). Pharmacological inhibition of gut-derived serotonin synthesis is a potential bone anabolic treatment for osteoporosis. Nat. Med. 16, 308-312.

Yadav, V. K., Ryu, J. H., Suda, N., Tanaka, K. F., Gingrich, J. A., Schutz, G., Glorieux, F. H., Chiang, C. Y., Zajac, J. D., Insogna, K. L., Mann, J. J., Hen, R., Ducy, P., and Karsenty, G. (2008). Lrp5 controls bone formation by inhibiting serotonin synthesis in the duodenum. Cell 135, 825-837.

Received: 27 October 2010; accepted: 23 November 2010; published online: 10 December 2010.

Citation: Tobias J (2010) Grand challenges in bone endocrinology. Front. Endocrin. 1:8. doi: 10.3389/ fendo.2010.00008

This article was submitted to Frontiers in Bone Endocrinology, a specialty of Frontiers in Endocrinology.

Copyright (c) 2010 Tobias. This is an open-access article subject to an exclusive license agreement between the authors and the Frontiers Research Foundation, which permits unrestricted use, distribution, and reproduction in any medium, provided the original authors and source are credited. 